\title{
A Knowledge-Intensive Decision Support System for Industrial Machines Maintenance
}

\author{
DJAMILA BOUHALOUAN, BAKHTA NACHET, ABDELKADER ADLA \\ Department of Computer Science \\ University of Oran 1 Ahmed Ben Bella \\ Oran, ALGERIA \\ adla.abdelkader@univ-oran1.dz
}

\begin{abstract}
In industrial plants, the profitability of the plant is significantly affected by the quality of machines maintenance. To ensure continuous production, the high valued machines should be kept in good working conditions. This brings plants to search for means to control and reduce equipment failures. When faults emerge in plants, appropriate actions for fault diagnosis and troubleshooting must be executed promptly and effectively to prevent large costs due to breakdowns. To provide reliable and effective maintenance support, the aid of advanced decision support technology utilizing previous repair experience is of crucial importance for the expert operators as it provides them valuable troubleshooting clues for new faults. Artificial intelligence (AI) technology, particularly, knowledge-based approach is promising for this domain. It captures efficiency of problem solving expertise from the domain experts; guides the expert operators in rapid fault detection and troubleshooting. This paper focuses on the design and development of a Knowledge-Intensive Decision Support System (KI-DSS) for Maintenance, Repair and Service in industrial plants to support better maintenance decision and improve maintenance efficiency. With integration of case-based Reasoning and ontology, the Ki- DSS not only carries out data matching retrieval, but also performs semantic associated data access which is important for intelligent knowledge retrieval in decision support system. A case is executed to illustrate the use of the proposed KI-DSS to show the feasibility of our approach and the benefit of the ontology support.
\end{abstract}

Keywords: -Decision Support Systems, Knowledge-Intensive DSS, Case-Based Reasoning, Ontology, Owl, Maintenance, Diagnosis

Received: October 29, 2019. Revised: March 15, 2020. Accepted: April 2, 2020. Published: April 13, 2020.

\section{Introduction}

Knowledge capitalization is of a considerable contribution during organizational problem solving activities which are often critical and recurring in nature. The development of a shared memory that stores the knowledge of expert members and their experience invoked in prior solutions can be useful for expert decision makers engaged in similar problem solving activities and will clearly assist them [1].

For an expert decision maker, it would be easier to reuse solutions and resolution schema corresponding to similar problems that have worked in the past than to analyze and solve the problems in scratch. Therefore, mechanisms to capture the experiential knowledge of experts can be of significant value to the organization in general, and the decision makers in particular.

A knowledge based approach shows significant promise for improving the effectiveness of maintenance activities support. It allows accumulating, organizing, storing, and sharing knowledge coming from past experience [2].
Among existing AI technology, Case-based reasoning (CBR) as an alternative reasoning paradigm and computational problem solving method has increasingly attracted more and more attention and grown in importance for businesses and academics over the past few years. The main principle of CBR is: similar problems have similar solutions. But, existing CBR systems lack semantic understanding, which is important for intelligent knowledge retrieval in knowledge-based systems [3]. To overcome this drawback, ontology technology is an ideal selection for realizing knowledge-based systems because ontology has not only powerful ability of knowledge representation, but also good semantic understanding. However, the explicit use of ontology based reasoning to support repetitive problem solving activities has received less attention.

To develop a such effective systems, two issues are critical: the first is how to find an effective method for case representation, which ensures domain knowledge can be acquired in an accurate easy manner, thus laying a good foundation for case retrieval; the next is how to find an 
appropriate method for case retrieval, which assures the right knowledge can be retrieved to solve a specific problem when a new task takes place. The system can not only carry out data matching retrieval, but also perform semantic associated data access, and improve the traditional keyword-based search. Through the semantic search capability, the hidden, but previously defined relations among data and concepts could be shown and represented if needed. These relations allow the user to understand the knowledge behind the stored data. Semantic technology could lead to the improvement in knowledge extraction, dissemination and management. Moreover, the use of common and unified domain ontology can improve the problem solving process where most of the decisions are dependent on individual experiences and domain knowledge of relevant managerial personnel.

The objective of this paper is to construct a maintenance intelligent knowledge-based system that can leverage the support of semantics. We suggest that the integration of ontology and CBR within a knowledge-based system is likely to provide additional information processing support. Ontology is used as a means to acquire domain knowledge and construct a case-base and use ontological semantic retrieval method as the case retrieval. Besides the case base, the system uses ontology (domain and task ontology related to the combustion machines to be maintained. This system will allow a more efficient searching in the case base by exploiting the semantic relations which exist between the cases. The system uses the semantic relations existing between the concepts within each of the ontology.

We experiment our system in maintenance domain, a semi-structured problem solving environment involving multiple attributes.

The remaining part of the paper is organized as follows. First, we present the case study related to maintenance in industrial plants. Then, we outline the related work on knowledge systems using ontology and case-based reasoning. Next, we present our Knowledge-Intensive Decision Support System. Finally, we present an example relating to maintenance of industrial machine before concluding

\section{Case Study}

In industrial plants, the presence of abnormal events generates risks. To avoid such risks, safety barriers are set up. However, barriers may not work properly, and thus abnormal events may arise. In such a case, industrial technicians intervene to diagnose the failures basing mostly on past failure experiences occurred in similar situations.

We apply our approach in maintenance domain. Maintenance deals with the detection and isolation of abnormal events. It consists of interpreting the current state of the machine from sensor readings and process knowledge. Maintenance is of crucial importance in terms of safety and also of economics, because of the influence of abnormal events in yield and quality of products.

A fault may be defined as an abnormal change in the characteristics of a system which gives rise to undesirable performance. Equivalently, the change of performance could be due to performance deterioration or malfunction. Hence, the operators should know the cause(s) of the change. In such cases the diagnosis tool should be able to identify the cause(s).

The application concerns EMETAL; an Algerian company which offers a wide range of sheet bending machines built for years of operation at full capacity. The machines are manufactured for the automotive industry, outdoor and indoor furniture, supermarkets and household electrical appliances, etc. The machines are numerically controlled tools that bend and fold sheets and tubes, precision and industrial sheet metal, steel sheet and strip. The controllers perform a variety of functions including protecting the machine from damage by performing an automated shutdown when dangerous conditions are detected and archiving sensor data.

As of 2016 there are thousands of these machines in use by EMETAL's customers national wide. EMETAL has contracts to service more than a thousand of machines and that number has been growing by hundreds every year. Different types of machines are manufactured in this company. Each machine is identified with two parameters: the number of axes and the diameter of the tube to be bent. The FS Series machine is a folding machine with three axes: feeding, folding and folding. This machine can bend wires with diameters up to 7 $\mathrm{mm}$. The FS Series machine is a combination of 13 different components. The Parvex drive in the switch cabinet is a particularly critical component. The Parvex drive consists of a set of drives.

A statistical study was carried out on the failures encountered in the company. Some of the failures are common between the different machines. In most cases, the failure is due to the electrical problem. The Parvex drive is the component the most affected by the failures. The duration of repairing can last up to a few weeks for complex problems. 
Currently, the machine maintenance process was as follows. When a machine has broken down or a malfunction of a machine is detected, the onsite operator will call the company and will send a message. The breakdown will be assigned to a company technician for analysis. The technicians take into account the information provided by the customer, will access the data from the machine, review key values, draw on their previous knowhow and experience, create a hypothesis about the breakdown cause, create plots specific to the breakdown type hypothesized, confirm the cause of the breakdown as best as can be done using the available data, then call the site to provide assistance and confirm the breakdown cause. Each repairing is recorded. The saved records are not well formalized and thus unexploited. These sheets should be used as a basis of experience for use in future repairs.

The goals are to improve machine and system reliability, reduced machine operating/maintenance costs, and produce the greatest possible sustained availability from the machine. For the company, it is a matter of better formalizing the experiences of the experts on maintenance, using reasoning from experience. The knowledge-based approach is to be used to automate the data review, hypothesis generation, and hypothesis confirmation of this process whenever possible and assist the user when it does not have confidence in a single cause.

\section{Related Work}

Knowledge management encompasses various practices of managing knowledge such as knowledge generation, capture, sharing, and application. Within these practices, effective sharing and use of knowledge depends - to a large extent - on the organization's ability to create and manage its knowledge. This knowledge can be described as the way organizations store it from the past to support present activities [4].

Knowledge management and Case-Based Reasoning are two intertwined topics. Case-based reasoning is a problem solving paradigm that in many respects is fundamentally different from other major AI approaches. Instead of relying solely on general knowledge of a problem domain, or making associations along generalized relationships between problem descriptors and conclusions, the case-based reasoning formalism was proposed as a way of storing human experiences and retrieving stored cases similar to the current item through a process of analogical search. It draws its knowledge from a reasonably large set of cases contained in the case library of past problems and by adapting their solutions solves new problems rather than only from a set of rules. Furthermore, case-based reasoning systems are claimed to "learn" through addition of further significant cases to the case-base and by forms of abstraction which may then be applied to this collection of cases [5].

Reasoning by re-using past cases is a powerful and frequently applied way to solve problems for humans. However, one of the drawbacks of CBR is the lack of flexibility of the knowledge representation. Indeed, the structure of the case is considered as constraining and strict which does not allow dealing with a carried out experiment in its semantic context, really limiting the performances of the system. As a way to deal with needs, ontology technology is an ideal selection for realizing knowledge-based decision support systems because ontology has not only powerful ability of knowledge representation, but also good semantic understanding. Ontologies provide a semantic based approach to explicitly represent information in a computable manner so that information can be automatically processed and integrated. Ontology also provides shared understanding of a domain to overcome differences in terminology from various sources [6]. The integration of an ontology-based model and CBR within a knowledge-based system has its advantages in: (1) Facilitating knowledge sharing by providing a formal specification of the semantics for context information; (2) Supporting for logic reasoning, referring to the capability of inferring new context information based on the defined classes and properties; (3) Enabling knowledge reuse by use of existing and mature ontology libraries without starting from scratch; (4) Having the stronger ability for expressing complex context information.

Several studies have given empirical evidence for the dominating role of ontologies integrated with specific, previously experienced situations (what we call cases) in human problem solving. Park and his colleagues [7] propose an ontology-based fuzzy CBR support system for ship's collision avoidance to prevent the cumbersome tasks of creating a new solution each time a new situation is encountered. A case-based decision support system applied to loan evaluation is developed in [8]. The approach uses AHP method to select important features and fuzzy sets technique to measure similarity between cases. A proposal presented in [3] aimed at knowledge reuse, during the decision activities by means of 
interwoven concepts from the knowledge management research. In [9] the constructed decision support CBR prototype system of marketing strategy contains more than 600 cases. The evaluation shows that with the support of semantics, they can not only carry out data matching retrieval, but also perform semantic associated data access. Kobti and Chen [10] construct domain ontology of mold design and propose an ontology-based search model to improve the traditional keyword-based search for the mold design domain. In [11], the authors proposed an approach based on the integration of three techniques: a CBR-personalized retrieval mechanism designed to provide a user with an optimum itinerary that meets his personal needs and preferences; a semantic web rule language considered to provide the system with enhanced semantic capabilities and support personalized case representation; and a user-oriented ontology used as source of knowledge to extract pertinent information about stakeholder's preferences and needs. To facilitate decision making within collaborative design, a Decision Support Ontology (DSO) is developed in [12]. The structure of the information model developed reflects a priori knowledge of decision making and supports the communication of information independent of any specific decision method. A case-based reasoning (CBR) system for the Semantic Web is presented in [13]. It implements a generic case-based inference mechanism in which adaptation consists in retrieving similar cases and in replacing some features of these cases in order to obtain one or more solutions for a given query. In [14] the authors propose a knowledge base for the Process Equipment Failures (PEFs) through semantic feature, embedded in the ontological approach and to construct a base frame for its further applications in the PEFs and process equipment related incident investigations and other knowledge extraction processes. A knowledge-based approach to support decision making in human resource management is proposed in [15]. The appropriate support of decision making is implemented using case-based reasoning and ontology. The problems of knowledge and case representation are considered, as well as the algorithm of case retrieval. Among other systems we cite the platform PROTEUS [16], and the Risks Analysis Support Tool in industrial domain [17].

Many research efforts for decision modelling and support have been systematically applied to the field of ontologies. However, there is no complete method that would define how to model decisions in ontologies, and a few isolated cases in which an established decision making method was used in ontology for a specific domain, and often the reasoning procedure is based only on domain ontology.

In our approach, we consider particularly the case where the reasoning process is enriched by exploring ontology. Thereby the purpose is to retrieve and provide a set of possible solutions relating to source case showing the semantic relations between them. Afterwards, it is the duty of the decision-maker, according to his/her expertise, to opt for the decision which will seem to him appropriate to the target problem. An important goal of our work was to structure decision model in such a way that the problem solution can be obtained by reasoning upon three ontologies (domain, task, and decision). The ontologies with reasoning support can be used in the function of a case base reasoning system.

\section{The Knowledge-Intensive Decision Support System}

The frame of our work is to integrate a knowledgebased tool in a Group Decision Support System (GDSS) that will be exploited by the actors (decision makers) for the purpose of decision support [18][19]. . The proposed system will assist the actors involved in maintenance session by offering them a set of decisions for the new problem and it is for the actor to situate each solution in its semantic context and then choose a particular solution based on his expertise.

We are in the context where typically incidents are not entirely identical to each other (some symptoms are not observed) but the knowledge of past incidents enables decision makers to recognize a similar situation and tailor their strategies by taking a course of action that experience has shown is effective and successful. This can happen when there is failure at some sensors so that lights or alarms cannot be triggered. The search in the database of cases can then be disoriented.

The benefits of using the system is to provide a more convenient retrieving process in information retrieval system in order to reach conclusions and give recommendations based on knowledge from previous cases (experiences) and ontologies.

A knowledge-based problem-solving approach adopting CBR is used to solve a new problem (target case) by remembering a previous similar situation (source case) and by reusing information and knowledge of that situation. The effectiveness 
of this approach is further improved by the application of ontologies as a mechanism for reasoning about the domain concepts and dealing with the inconsistencies that can arise in the applied vocabulary when multiple decision makers are involved. Thus, our approach to knowledge-based systems is towards integrated applications that combine case specific knowledge with models of general domain knowledge. The more knowledge is embedded into the system, the more effective is expected to be. Semantic CBR processes can take advantage of this domain knowledge and obtain more accurate results.

\subsection{The Case Base}

Knowledge representation is essential in building a knowledge-based system since on this presentation depends the effectiveness and the fastness of the system case retrieving mechanism. It is therefore necessary to well identify information to be stored in each case and to choose the more efficient representation scheme of this information. A case is a contextualized piece of knowledge representing an experience. The information encoded about the past experiences, depends on the domain of application as well as on the goal for which the cases are used. Case indexing and storage are an important aspect in designing efficient knowledgebased decision support systems in that, it should reflect the conceptual view of what is represented in the case and take into account the indices that characterize the case.

Knowledge considered in our knowledge-based decision support system is represented by cases and ontologies. The case base is composed of all the structured cases which will be explored during retrieving step (recall stage). Every case consists of a breakdown problem already experienced and solved. A case represents a diagnosis experience, and thus consists of two main parts: a problem part describing the failure, and a solution part. Each part is represented by a set of simple or complex descriptors among which some are defined in an ontology.

Problem part: the task to be solved;

Solution part: the solution, the problem solving method used and, the object concerned by recommended solution

The case base is a finite set of source cases (S), denoted by $\mathrm{CB}=\{\mathrm{S} 1, \mathrm{~S} 2, \ldots \mathrm{Sn}\}$ where a source case $\mathrm{Si}=(\mathrm{PbS}, \mathrm{Sol}[\mathrm{PbS}]) / \mathrm{PbS}$ is the source problem part and $\mathrm{Sol}[\mathrm{PbS}]$ is the solution part of the source case. Sol $[\mathrm{PbS}]=\{[\mathrm{A} 1 / \mathrm{V} 1],[\mathrm{A} 2 / \mathrm{V} 2], \ldots$ $[\mathrm{An} / \mathrm{Vn}]\}$ where $[\mathrm{Ai} / \mathrm{Vi}]$ means [Attribute/Value]. The case base is created manually with typical failures. The target case (C) is denoted by $\mathrm{C}=$ $(\mathrm{PbC}$, Sol $[\mathrm{PbC}])$. The target case problem part $(\mathrm{PbC})$ is the structural representation of the new failure to be repaired; the solution of the target case $(\mathrm{Sol}[\mathrm{PbC}])$ is the structural representation of the resolution of this failure. The attributes of the $\mathrm{PbC}$ are filled upon an analysis phase of the problem parameters introduced by the user. This analysis involves the task ontology and allows structurally representing the new problem to be solved. Initially, the attributes of the $\mathrm{Sol}(\mathrm{PbC})$ part remain empty. Fig. 1 shows the UML classes diagram relating to the modelling of the case base. The descriptors are entries to the ontologies (e.g. IdTask, Id-Symp and Id-Cause are entries for the task ontology; the descriptors Id-Object is an entry for the domain ontology, and the descriptor IdSolution-Id is an entry for the application ontology).

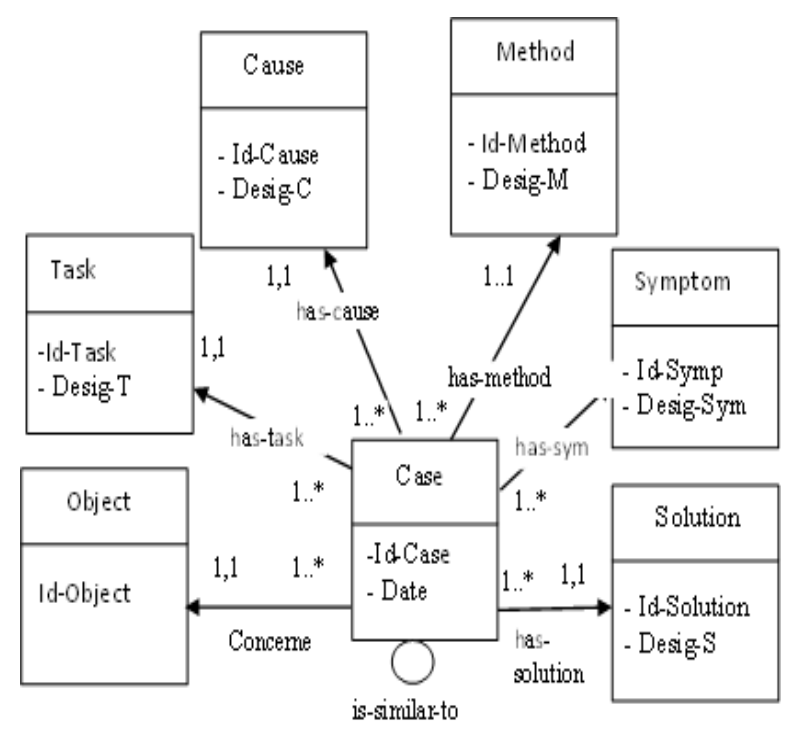

Fig. 1: UML Class Diagram of the Case Base

\subsection{Ontology Modelling}

\subsubsection{Conceptualization}

The ontology development methodology is usually composed of several strategies on defining classes and class hierarchy, defining properties and naming considerations. We used the METHONTOLOGY method [20] to build the ontology.

The ontology is created based on documentation resources as all the potential decisions that might be made by the decision makers are listed in an appropriate documentation. Similarly, the 
description of the equipment to be maintained is get from specific documentation while the specification of the task ontology is built with the support of an expert in industrial maintenance.

The ontology is composed of three parts related to equipment domain, maintenance task and application.

Fig. 2 present UML classes diagram of the proposed ontology. The equipment domain part consists of a specification of the concepts relating to the equipment to maintain as well as the relations between these concepts. The latter are principally aggregation and composition relations between the equipment components. The task part described all the maintenance problems related to the combustion machine (equipment) in terms of task, symptom, cause and solution concepts, and the relations between them while the application domain part represents the domain of decisions.
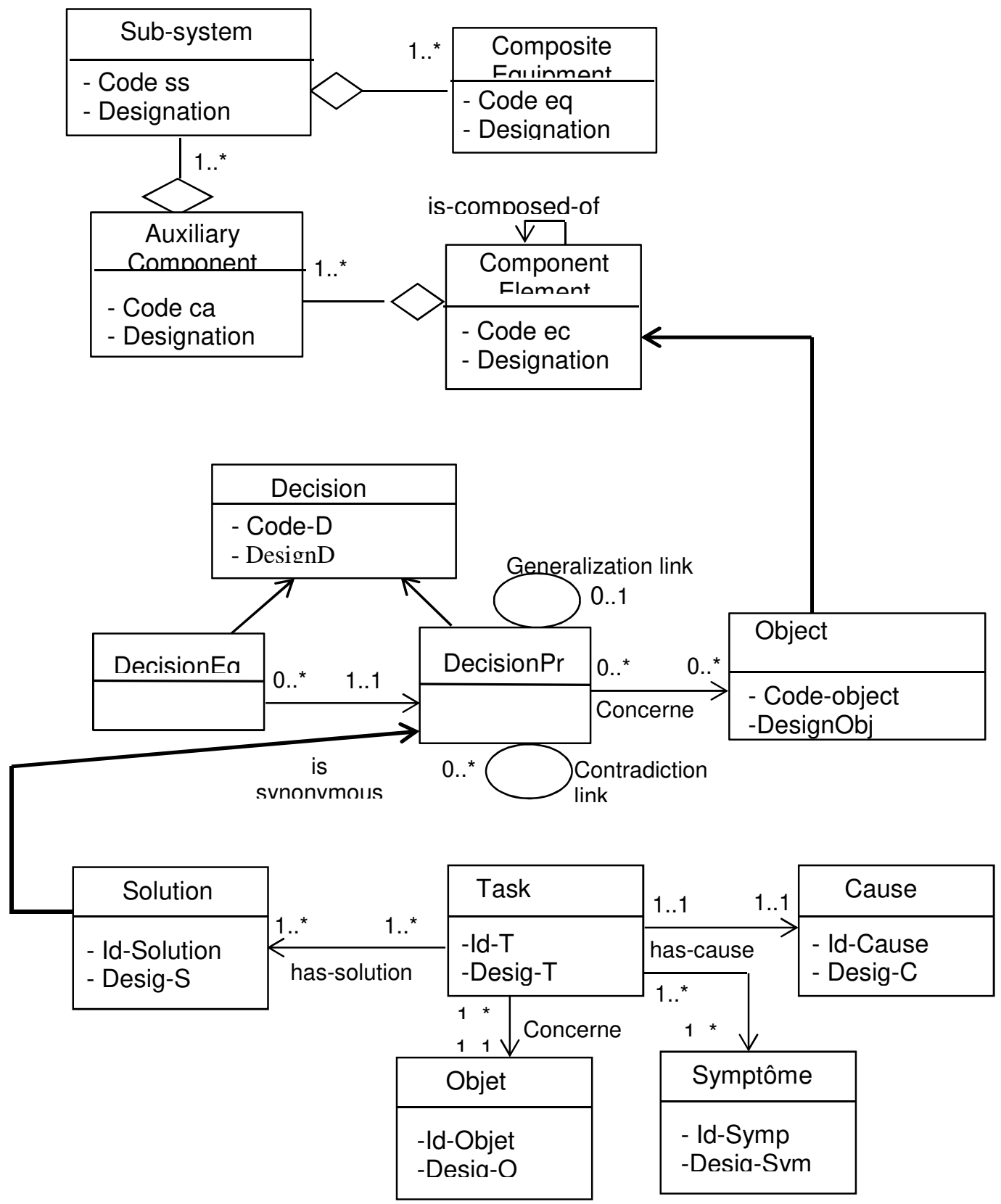

Fig. 2: Conceptual model of the ontology 


\subsubsection{Conceptualization}

The ontology is created using Protégé before their generation in OWL format [21]. Fig. 3 illustrates a partial view of the ontology.

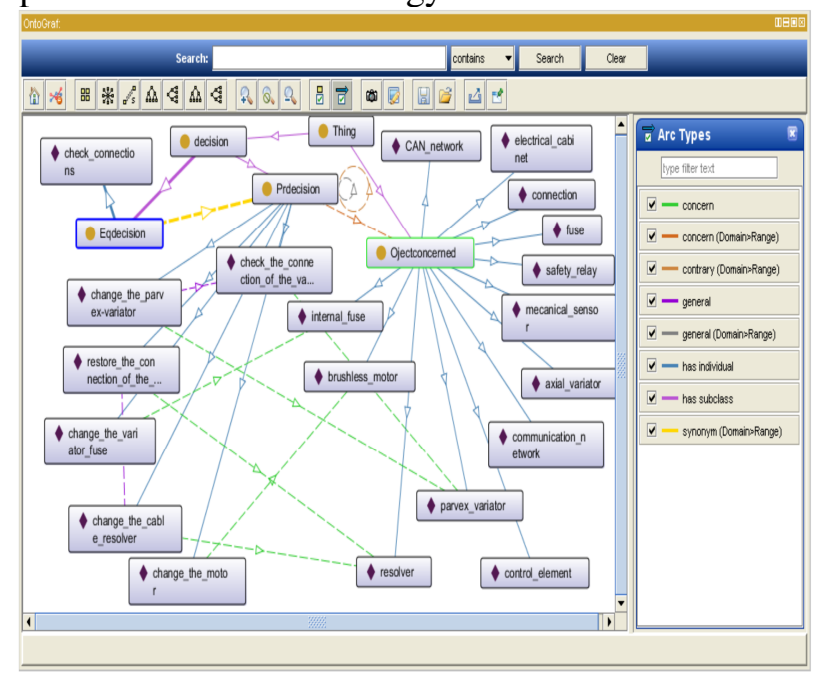

Fig. 5: Partial view of the decision ontology

\subsubsection{Operationalization}

An operationalized ontology is expressed in an operational language and endowed with operational semantics. In this sense the ontology operationalization consists of a computer specification of all the operations made on concepts in an operational language. The use of an operational ontology assumes its representation in an operational but also formal language, i.e. providing reasoning mechanisms appropriate to the targeted knowledge manipulations. To do this, we used the NetBeans developing environment associated to Java language [22]. Furthermore, we used the Jena framework Jena [23] to manage the ontology. Jena provides a programming environment for RDF, RDFS [24]) and OWL as well as a query engine allowing SPARQL queries execution (Simple Protocol And RDF Query Language) [25] which is a RDF query language.

Table 1: Example of the source case T3

<owl:NamedIndividual rdf:about="http://www.basedecas.org/ontologycases\# 3">

$<$ rdf:type rdf:resource="http:// www.basedecas.org/ontologycases\#Cases"/>

$<$ rdf:type rdf:resource="http:// www.basedecas.org/ontologycases\#Cases"/>

$<$ has-as-task rdf:resource="http:// www.basedecas.org/ontologycases\#T3"/>

$<$ has-as-cause rdf:resource="http:// www.basedecas.org/ontologycases\# Failure to turn up the variator"/ $>$

$<$ has-as-method rdf:resource="http://www.basedecas.org/ontologycases\#M1"/>

$<$ concerns rdf:resource="http://www.basedecas.org/ontologycases\# Internal fuse"/>

$<$ has-as-solution rdf:resource="http://www.basedecas.org/ontologycases\#change internal fuse"/>

$<$ possesses rdf:resource="http://www.basedecas.org/ontologycases\#display variator off"/>

$<$ possesses rdf:resource="http://www.basedecas.org/ontologycases\# Failure to turn up the variator, the machine is shut down"/>

$<$ is-similar-tordf:resource="http://www.basedecas.org/ontologycases\#15"/>

$<$ owl:NamedIndividual $>$

OWL language [21] is used to represent the case base. This would allow managing the case base as a knowledge base upon which inferences may be made. It is possible to define semantic relations between cases as for instance the transitive relation "is-similar-to" which relates the source cases already identified as being similar. Furthermore, as the remained knowledge (i.e. the ontologies) is also expressed in OWL, this would allow having to some extent compatibility between languages formalizing the different knowledge manipulated by the system, as well as, the knowledge operating tools such as SPARQL.

\subsection{The Reasoning Process}

The proposed case based system should reflect human knowledge by storing data about previous significant events as "cases" within a computerized system. In this regard, the system uses the case base to retrieve similar cases to the problem to be solved. But, when the retrieving process fails or the cases retrieved are not satisfactory for the decision maker, the system uses ontologies. It makes use semantic relations between concepts within the same ontology to derive other solutions to the problem.

By making use of the ontology, the system derives more specific or more general decisions than those initially retrieved by the system. It can also set the solution relating to the equipment by visualizing the concerned component. Then, it uses the ontology to set the involved component relating to the neighboring ones or to the component in which it's comprised. Similarly other case 
descriptors may be used as entries to the ontology to enlarge or reduce solution space. When a solution is retained, then tested and validated, it is stored in the case base as a new case (with all its descriptors).

The reasoning process consists of the following steps (Fig. 4):

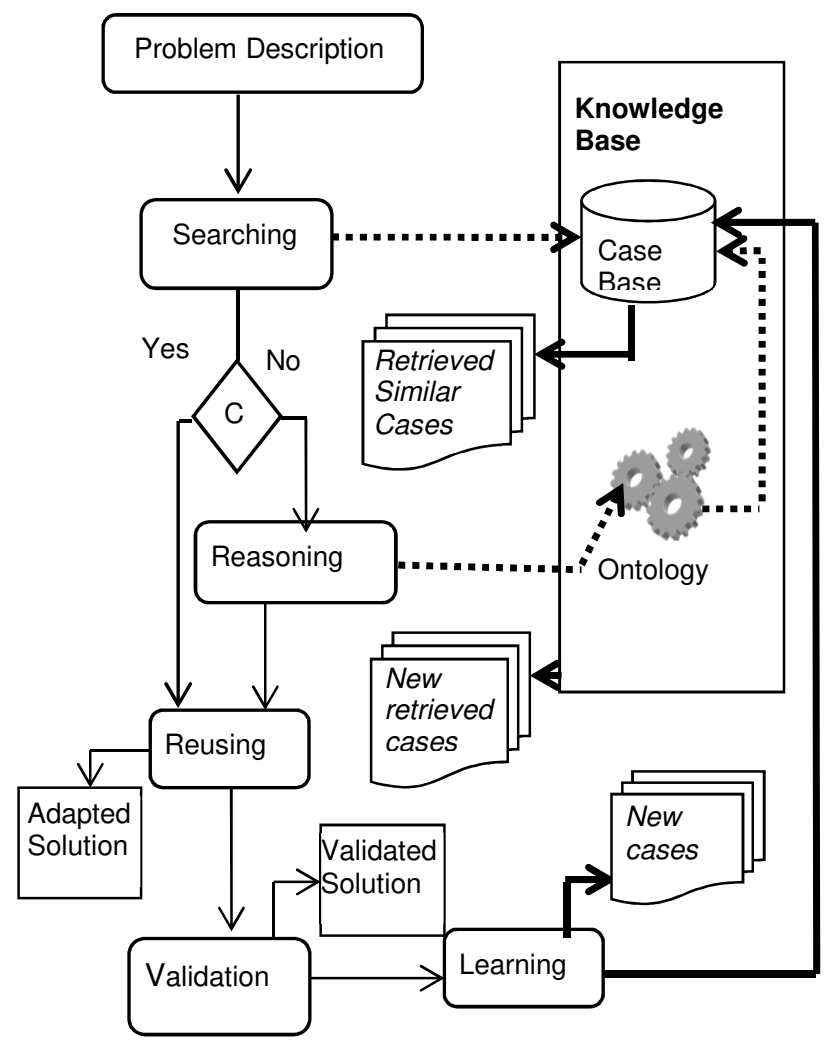

$\mathrm{C}$ : Is the result satisfactory?

Fig. 4: Ontology-based CBR Process

Problem description: The decision maker describes the problem to be solved. This description can be made of different ways: by providing the task to be solved, the observed symptoms, or the faulty object, etc.

Retrieving: It consists to search in the case base and retrieve similar cases to the problem to be solved. Here, we consider the usual local and global similarities measures to retrieve similar cases to the targeted problem. Once the target problem is introduced, the goal of this step is to recall the source case that is most similar to the target case, applying two measures of similarity (local, global) between the target problem and the source problem.

Local Similarity: This similarity measure is computed between the value of an attribute in target problem $(\mathrm{PbC})$ and the value of the same attribute in source problem $(\mathrm{PbS})$. It is evaluated in two different ways depending on whether the attribute is simple or complex.

Simple attribute: The simple attribute in our case is the symptom attribute which has a unique value. The local similarity between the attributes is equal to 1 if the values of the two attributes are equal (see equation (1)), else it is equal to 0 .

$$
\operatorname{Sim}_{\mathrm{i}}\left(\mathrm{s}_{\mathrm{i}}, \mathrm{c}_{\mathrm{i}}\right)=\left\{\begin{array}{l}
1 \text { pour } c i=s i \\
0 \text { pour } c i \neq s \mathrm{si}
\end{array}\right.
$$

$\mathrm{C}_{\mathrm{i}}$ : Value of the attribute $i$ in $\mathrm{PbC}$ part.

$\mathrm{S}_{\mathrm{i}}$ : Value of the same attribute $i \mathrm{PbS}$ part.

Complex Attributes: The complex attribute in our case is the symptom attribute which has a list of values. The local similarity between the values of the symptom attribute of the $\mathrm{PbC}$ and the values of the same symptom attribute of the $\mathrm{PbS}$ is given by equation 2 below:

$$
\operatorname{sim}(\mathrm{si}, \mathrm{ci})=\frac{n i * 2}{n 1+n 2}
$$

ni: number of equal values in both target and source problem for the $i$ attribute.

$\mathrm{n} 1$ : number of values relating to the attribute in the source problem.

$\mathrm{n} 2$ : number of values relating to the attribute in the target problem.

Global similarity: This similarity measure is calculated between a set of attributes in the $\mathrm{PbC}$ and the same set of attributes in the $\mathrm{PbS}$, it corresponds to the mean of the local similarities and its value is in the range [0,1 ]. It is defined by the similarity function below:

$$
\operatorname{SIM}(\mathrm{S}, \mathrm{C})=\frac{1}{n}+\sum_{i=1}^{n} \operatorname{simi}(\mathrm{si}, \mathrm{ci})
$$

\section{C: target case.}

S: source case.

$\mathrm{n}$ : number of attributes in which the local similarity has been calculated.

$\operatorname{sim}_{\mathrm{i}}\left(\mathrm{s}_{\mathrm{i}}, \mathrm{c}_{\mathrm{i}}\right)$ : value of local similarity for attribute $i$.

To search a $\mathrm{PbS}$, the most similar to the $\mathrm{PbC}$. For this, we apply the measure of local then global similarities. In the global similarity measure, a similarity threshold is determined at 0.5. If SIM (S, C) $<0.5$ the source case is considered negligible (not similar) else if SIM (S, C) $\geq 0.5$ the source case is considered important (partially similar). In 
case $\operatorname{SIM}(\mathrm{S}, \mathrm{C})=1$ the source case is considered perfectly similar.

Reasoning: If a perfectly similar case is not found, we move on ontology-based reasoning in order to search other source cases. For each of the initially retrieved source cases, we use semantic relations between the source cases to derive other source cases semantically close to similar cases retrieved. These if they exist are presented to the user. The latter, if the suggested solutions are satisfactory, the process of recalling is stopped and we pass to the next phase of the reasoning process. Furthermore The ontology may be used to enlarge or to reduce the solution search space. According to the object of widening, the ontology is used. For example when the object of widening is a task or a symptom, the task ontology part is used; when the object of widening is a faulty component, the equipment domain ontology part is explored, but when the object of enlargement is the problem solution then the decision ontology part is used.

Validation: Once the decision is made, executed and validated the process will skip to the next step.

Learning: The new case is added to the case base. It referees to all the similar source cases if exist.

The reasoning step is useful as it allows revealing semantic knowledge from ontologies between the different parameters of the problem to be solved. Given a problem to be solved, this would allow: 1) Converging to the semantically close case in the case base, or 2) Retrieving first a structurally close case from the case base then, according to the case descriptors, exploiting ontologies in order to derive other possible solutions to the problem. The decision-maker will choose among the suggested solutions that he considers being the most appropriate one to the problem.

\section{Application}

In order to contribute, we determine in a base of reference cases (maps of problem solving and their solutions), which cases are closest or similar to that studied or to be treated. Business experts will be able to study these analogous or similar cases in order to deliver their diagnosis and propose a repair. This case base will represent a tool for decision-making when solving future industrial problems.

The objectives are to reduce the diagnosing and problem solving time, quickly analyze the breakdown, provide technicians with tools to help them be more effective at the diagnosis stage, and to transfer knowledge on unformed trades and train people for more efficiency and performance. The company wants to reduce the diagnostic time for more frequent breakdowns.

Table 2:The case base

\begin{tabular}{|l|l|l|l|l|l|}
\hline $\begin{array}{l}\text { Id- } \\
\text { T }\end{array}$ & Task & Cause & Method & Object & Solution \\
\hline 1 & T1 & Resolver break failure & M3 & $\begin{array}{l}\text { Resolver } \\
\text { cable }\end{array}$ & Check the resolver cable \\
\hline 2 & T2 & $\begin{array}{l}\text { Breaking of one or more wires of the } \\
\text { encoder }\end{array}$ & M2 & Encoder & Changing the encoder \\
\hline 3 & T3 & Failure to power up the variator & M1 & Internal fuse & Change the internal fuse \\
\hline 4 & T4 & Internal drive failure & M4 & Drive & Changing the drive \\
\hline 5 & T5 & High temperature & M6 & Radiator & Clean the radiator \\
\hline 6 & T6 & Excessive engine speed & M5 & Engine & Changing engine \\
\hline 7 & T7 & Drive power incident & M1 & Internal fuse & $\begin{array}{l}\text { Changing the internal } \\
\text { fuse }\end{array}$ \\
\hline$\ldots$ & $\ldots$ & $\ldots$ & $\ldots$ & $\ldots$ & $\ldots$ \\
\hline
\end{tabular}


Table 3: List of symptoms

\begin{tabular}{|l|l|}
\hline Id-T & Symptoms \\
\hline 1 & Symp 1(Failure to power up the machine, the variator is shut down) \\
\hline 1 & Symp 3 (Fault 2 appears on the axis drive display) \\
\hline 2 & Symp 2 (No information at the encoder display) \\
\hline 3 & Symp 1(Failure to power up the machine, the variator is shut down) \\
\hline 3 & Symp 4 (At the time of automatic start the orientation head moves downwards) \\
\hline 3 & Symp 10 (Fault 4 appears on the display of the axis drive) \\
\hline 3 & Symp 18 (No information displayed) \\
\hline 3 & Symp 20 (Electrical axis incident, drive power incident) \\
\hline 4 & Symp 1 (Failure to turn up the machine, the variator is shut down) \\
\hline 4 & Symp 20 (Electrical axis incident, drive power incident) \\
\hline 4 & Symp 21 (Absence of "READY" (Power E1)) (Symp displayed on the computer screen) \\
\hline 5 & Symp 7 (Fault 4 appears on the display of the axis drive) \\
\hline 5 & Symp 20 (Electrical axis incident, drive power incident) \\
\hline 5 & Symp 21 (absence of "READY" (Power E1)) (Symp displayed on the computer screen) \\
\hline 6 & Symp 9 (High engine temperature) \\
\hline 6 & Symp 18 (No information displayed) \\
\hline 6 & Symp 1 (Failure to power up the machine, the variator is shut down) \\
\hline$\ldots$ & $\ldots$ \\
\hline
\end{tabular}

Let consider the following description of the problem: "Variator failure". This problem is characterized by the following symptoms: "Symp1 (Symp1: impossible to power up the variator), Symp18 (No information on the drive displayed). We do search for similar cases to the problem occurred.

\begin{tabular}{|l|l|l|}
\hline \multirow{4}{*}{$\begin{array}{l}\text { New } \\
\text { Problem }\end{array}$} & Task & Variator failure \\
\cline { 3 - 3 } & Cause & \\
\cline { 3 - 3 } & symptoms & $\begin{array}{l}\text { Impossible to } \\
\text { power up the } \\
\text { variator }\end{array}$ \\
\cline { 3 - 3 } & $\begin{array}{l}\text { No information on } \\
\text { the drive displayed }\end{array}$ \\
\hline $\begin{array}{l}\text { New } \\
\text { Problem } \\
\text { Solution }\end{array}$ & Method & \\
\cline { 2 - 3 } & Objet & \\
\cline { 2 - 3 } & Solution & \\
\hline
\end{tabular}

The task ontology is used to infer the features lacking (Cause) in the target problem structure.

\begin{tabular}{|c|c|c|}
\hline \multirow{4}{*}{$\begin{array}{l}\text { New } \\
\text { Problem }\end{array}$} & Task & Variator failure \\
\hline & \multirow{3}{*}{$\begin{array}{l}\text { Cause } \\
\text { symptoms }\end{array}$} & Drive power failure \\
\hline & & $\begin{array}{l}\text { Impossible to } \\
\text { power on the } \\
\text { machine }\end{array}$ \\
\hline & & $\begin{array}{l}\text { No information on } \\
\text { the drive displayed }\end{array}$ \\
\hline \multirow{3}{*}{$\begin{array}{l}\text { New } \\
\text { Problem } \\
\text { Solution }\end{array}$} & Method & \\
\hline & Object & \\
\hline & Solution & \\
\hline
\end{tabular}

We calculate the similarity measures between the attributes: task, cause, symptom.

\begin{tabular}{|c|c|c|c|c|}
\hline Case & \multicolumn{3}{|c|}{ Attributes } & \multirow{2}{*}{$\begin{array}{l}\text { Global } \\
\text { Similarity }\end{array}$} \\
\hline & Task & Cause & Symptom & \\
\hline $\mathrm{T} 1$ & 0 & 0 & 0.5 & 0.16 \\
\hline $\mathrm{T} 2$ & & & & \\
\hline T3 & 1 & 1 & 0.5 & 0.83 \\
\hline $\mathrm{T} 4$ & 0 & 1 & 0.4 & 0.46 \\
\hline
\end{tabular}

At the end of the second recall step, a source case $(\mathrm{PbS})$ similar to the task $\mathrm{T} 3$ is retrieved. If the user would accept the proposal, he will apply the Solution to the target case $(\mathrm{PbC})$.

\begin{tabular}{|l|l|l|}
\hline \multirow{3}{*}{$\begin{array}{l}\text { Similar saure } \\
\text { case }\end{array}$} & Id-T & 3 \\
\cline { 2 - 3 } & Task & T3 \\
\cline { 2 - 3 } & Cause & $\begin{array}{l}\text { Drive power } \\
\text { problem }\end{array}$ \\
\cline { 2 - 3 } & symptoms & Symp1 \\
\cline { 2 - 3 } & Symp2 \\
\hline \multirow{2}{*}{$\begin{array}{l}\text { Similar source } \\
\text { case solution }\end{array}$} & Method & M1 \\
\cline { 2 - 3 } & Object & Internal Fuse \\
\cline { 2 - 3 } & Solution & $\begin{array}{l}\text { Changing the } \\
\text { fuse }\end{array}$ \\
\hline
\end{tabular}

\section{Conclusion}

In this paper, we focused on the design and development of a Knowledge-Intensive Decision Support System (KI-DSS) to support better maintenance decision and improve maintenance 
efficiency in industrial plants. We integrated casebased reasoning and ontology. We claim that this combination is useful for the design of KI-DSS and strengthens its reasoning process as it allows the knowledge engineer to use knowledge already acquired, conceptualized and implemented in a formal language; reducing considerably the knowledge acquisition bottleneck.

The effectiveness of CBR can be further improved by the application of ontologies as a mechanism for reasoning about the domain concepts and dealing with the inconsistencies that can arise in the applied vocabulary when multiple decision makers are involved. Moreover, the reuse of ontologies from a library also benefits from their reliability and consistency.

We believe that our approach is useful in several aspects. First, it enables to formalize the case base in OWL what allows managing it as a knowledge base. Indeed, by exploiting the semantic relations within the case base, it is possible to derive new knowledge from those stored. Also, as a result of memorizing a source case base with its descriptors, the ontologies exploration will allow deriving new knowledge which will serve for a new research cycle in case base.

As future work, we aim to collect much information to use quantitative indices for performance evaluation of our approach. Further experiments are to be organized to evaluate the performance of our system with fault coverage rate, diagnosis effectiveness ratio, and other quantitative indices.

\section{References:}

[1] M. S. Ackerman, and C.A. Halverson, Reexamining organizational memory, Communications of the ACM, , 2001, pp. 58-64.

[2] A. Aamodt, and E. Plaza, Case-based reasoning: foundation issues, methodological variations and system approaches, Artificial Intelligence Communications, vol. 7, 1994, pp. $39-59$

[3] J. L. Garrido, M. V. Hurtado, M. Noguera, and J.M. Zurita, Using a CBR approach based on ontologies for recommendation and reuse of knowledge sharing in decision making, in Proceedings of 8th international conference on hybrid intelligent systems, 2008, pp. 837-42.

[4] B. Gallupe, Knowledge management systems: surveying the landscape, International Journal of Management Reviews, vol. 3, no 1, 2001, pp. 61-77.

[5] J. Kolodner, Case-Based Reasoning, Morgan Kaufmann Publishers, Inc, 1993.

[6] G. Antoniou, and F. Harmelen, A Semantic Web Primer, MIT, 2004.

[7] K. Park, R. M. Benedictos, C.S. Lee, and M. H. Wan, Ontology-based fuzzy-CBR support system for ship's collision avoidance, in Proceedings of the 6th international conference on machine learning and cybernetics, 2007.

[8] N. Benmessaoud, A. Adla, Intelligent Semantic Case Based Reasoning System for Fault Diagnosis, Journal of Digital Information Management (JDIM), Volume 17 Number 2, 2019.

[9] W. Dong, X. Yang, Z. Guobing, Z. Bo, Research on ontology-based case indexing in CBR in Shanghai, In: International conference on artificial intelligence and computational intelligence, ICAICI 09, IEEE Press, 2009, pp. $238-42$.

[10] Z. Kobti, and D. Chen, A domain ontology model for mold design automation, Canadian AI 2010, vol. 6085, 2010, pp. 336-9.

[11] J. A. Rockwell, I. R. Grosse, S. Krishnamurty, and J. C. Wileden, A Decision Support Ontology for collaborative decision making in engineering design, in Proceedings of Center for e-Design, 2009.

[12] A. Bouhana, A. Zidi, A. Fekih, H. Chabchoub, and M. Abed, An ontology-based CBR approach for personalized itinerary search systems for sustainable urban freight transport, Expert Systems with Applications, vol. 42, 2015, pp. 3724-3741.

[13] L. Lamontagne and E. Plaza, Tuuurbine: A Generic CBR Engine over RDFS, in proceedings of ICCBR, 2014, pp. 140-154.

[14] I. Mohammadfama, O. Kalatpour, R. Golmohammadi, and H. Khotanlou, Developing a process equipment failure knowledge base using ontology approach for process equipment related incident investigations, Journal of Loss Prevention in the Process Industries, vol. 26, 2013, pp. 1300-1307.

[15] M. Zhukova, M. Kultsova, A. Navrotsky, A. Dvoryankin, Intelligent Support of Decision Making in Human Resource Management Using Case-Based Reasoning and Ontology In Proceedings of JCKBSE 2014, pp. 172-184.

[16] I. Rasovsca. B. ChebelMorello. N. Zerhouni, A case elaboration methodology for a 
diagnostic and repair help system based on CBR, in " $20^{\text {th }}$ International Florida Artificial Intelligence Research Society Conference, FLAIRS'07., Key West, Florida (USA), 2007.

[17] B. Debray, C. Duval, C.A. Jovanovic, O. Salvi, Integrated management of emerging risks: challenges and objectives of the iNTegRisk European project, 16ème Congrès Lambda-Mu, Avignon, France, 2008.

[18] A. Adla, , J.L. Soubie, P. Zaraté, A cooperative Intelligent Decision Support System for Boilers Combustion Management based on a Distributed Architecture, Journal of Decision Systems (JDS), Vol. 16 No 2, 2007, pp. 241-263.

[19] A. Adla, P. Zarate, J.L. Soubie, A Proposal of ToolKit for GDSS Facilitators, Group Decision and Negotiation (GDN), Vol. 1, 2011.
[20] M. Fernandez, A. Gomez-Perez, N. Juristo, METHONTOLOGY: From Ontological Art Towards Ontological Engineering, In proceedings of the Ontological Engineering AAAI-97 Spring Symposium Series, 1997, Stanford University (USA), 1997.

[21] OWL 2 Web Ontology Language New Features and Rationale (Second Edition)W3C Recommendation 11 December 2012 http://www.w3.org/TR/owl-new-features/

[22] Java SE http://java.sun.com, 2012.

[23] Apache Jena http://jena.sourceforge.net/, 2012.

[24] RDF (Resource Description Framework) Model and Syntax Specification W3C Proposed Recommendation 05 January 1999 http://www.w3.org/TR/PR-rdf-syntax

[25] SPARQL Query Language for RDF W3C Recommendation 15 January 2008, http://www.w3.org/TR/rdf-sparql-query/ 